\title{
Added Value of Multitemporal Polarimetric UAVSAR Data for Permanent Scatterers Detection
}

\author{
Tina Nikaein ${ }^{1}$, Vahid Akbari ${ }^{2}$, Hossein Arefi ${ }^{1}$ \\ ${ }^{1}$ Department of Remote Sensing, School of Surveying and Geospatial Information Engineering, University of Tehran \\ ${ }^{2}$ Earth Observation Laboratory, Department of Physics and Technology, UiT The Arctic University of Norway
}

\begin{abstract}
In the last decades, differential synthetic aperture radar (SAR) interferometric (InSAR) (DInSAR) techniques have been used to estimate the Earth's surface deformation with high resolution. In this paper, we present an approach for increasing the quantity of permanent scattered pixels. These pixels are selected for DInSAR processing based on polarimetric information prepared by new sensors. The objective of this paper is then to test existing algorithms that confirm the contribution of polarimetric data for improving persistent scatterers (PS) detection. These algorithms are formulated based on two different selection criteria: amplitude dispersion index and mean coherence. Different approaches are analyzed to optimize both selection criteria in terms of pixels' quantity and density and finally their results are quantitatively compared. Experimental results with exploiting quad-pol UAVSAR data set over an urban area in $\mathrm{CA}$, provide the expected improvement. Comparing the number of PSs between quad-pol with dual-pol and single-pol cases illustrate remarkable improvement in both selection criteria. For quad-pol case, we achieve an increase of $50 \%$ and $60 \%$ with respect to dual-pol and single-pol data, respectively, when using average coherence and over 6 times more for amplitude dispersion index. The results of our study demonstrates the added value of polarimetric SAR observations (dual pol and quad-pol) for improved permanent scatterers detection monitored areas.

Index Terms-Uninhabited Aerial Vehicle Synthetic Aperture Radar (UAVSAR), Polarimetry, Permanent Scatterer, particle swarm optimization.
\end{abstract}

\section{INTRODUCTION}

The Earth's surface deformation is often caused due to landslide, subsidence, earthquake, and etc. that can be assessed using DInSAR techniques with high accuracy, high spatial resolution, and millimetric precision [1]. DInSAR has been used in the literature for mapping volcanic activity [2], monitoring seismic and tectonic deformation [3], [4], and ground subsidence monitoring [5]. All pixels of monitored area are not appropriate to use by classical DInSAR techniques. The quantity of reliable pixels in the studied area and their phase quality are important factors that can limit the efficiency of DInSAR techniques. In order to avoid decorrelation influence and other limiting factors in DInSAR processing, we need to identify reliable pixels to estimate deformation rate [6]. Permanent scatterer interferometry (PSI) technique has been extended the capability of traditional DInSAR methods as they process pixels that have partial decorrelations [7]. These approaches employ pixels which are stable during the data acquisition and choose only pixels that are known as a priori reliable considering certain quality criteria such as Amplitude Dispersion Index (ADI) [7] or average coherence [8], [9].
These pixels are referred as persistent scatterer (PS). In literature, PSI techniques applied on SAR data images that are usually acquired by the sensors providing single- channel data [7]-[11]. Polarimetric capability of current satellite sensors, such as Radarsat-2, ALOS-PALSAR, TerraSAR-X, and UAVSAR, makes it possible to have SAR data with more than one channel. In this paper, multitemporal quad-pol data sets from Jet Propulsion Laboratory/National Aeronautics and Space Administration (JPL/NASA)'s UAVSAR sensor are employed to analyze the capability of polarimetric data to increase the number of PSs. The proposed method includes in a search over the whole polarimetric space to optimize the pixel selection estimators. We analyze two estimators 1) mean coherence and 2) ADI that are formulated for quad-pol and dual-pol images.

This paper is organized as follows. In Section II, formulation of different methods to find optimum channel is presented. Used data sets to test all techniques are introduced in Section III. The obtained results with both estimators (i.e., ADI and mean coherence) and conclusion are summarized in Section IV and V, respectively.

\section{Methods}

A general formulation for polarimetric SAR interferometry was first proposed in [12]. In radar polarimetry, with vectorization of the scattering matrix $[\mathbf{S}]$ of each pixel, i.e.,

$$
\mathbf{S}=\left[\begin{array}{ll}
S_{h h} & S_{h v} \\
S_{v h} & S_{v v}
\end{array}\right]
$$

based on Pauli basis, the following scattering vector $\underline{k}$ is obtained under the assumption of the reciprocity theorem, i.e., $S_{h v}=S_{v h}$ :

$$
\underline{\mathbf{k}}_{i}=\frac{1}{\sqrt{2}}\left[S_{h h, i}+S_{v v, i}, S_{h h, i}-S_{v v, i}, 2 S_{h v, i}\right]^{T},
$$

where the subscript $i$ denotes the acquired PolSAR image at different times. $S_{h h}$ and $S_{v v}$ represent horizontal and vertical copolar channels, respectively and $S_{h v}$ stands for cross polar channel. The superscript $(\cdot)^{T}$ is vector transposition operator. According to [12], by projecting the Pauli scattering vector $\underline{\mathbf{k}}$ onto a unitary vector $\underline{\mathbf{w}}_{i}$, which can be parametrized by scattering mechanism angles, a scattering coefficient $\mu$ is generated as

$$
\mu_{i}=\underline{\mathbf{w}}^{H} \underline{\mathbf{k}}_{i},
$$


where $(\cdot)^{H}$ refers to the complex conjugate transpose. $\mu_{i}$ is a complex value including combination of all components of $\underline{\mathbf{k}}$ projected onto unitary vector $\underline{\mathbf{w}}_{i}$. Hence, it is similar to the new SAR images configuration to apply DInSAR processing to $\mu_{i} \mathrm{~s}$, similar to the single-pol data. $\underline{\mathbf{w}}_{i}$ can be parametrized for quad-pol data as in (4) and dual-pol data as in (5) [12] :

$$
\begin{gathered}
\underline{\mathbf{w}}_{i}=\left[\begin{array}{c}
\cos \alpha \\
\sin \alpha \cos \beta e^{j \delta} \\
\sin \alpha \sin \beta e^{j \gamma}
\end{array}\right] \begin{array}{c}
0 \leq \alpha \leq \frac{\pi}{2} \\
0 \leq \beta \leq \pi \\
0 \leq \delta \leq \pi \\
-\pi \leq \gamma \leq \pi
\end{array} \\
\underline{\mathbf{w}}_{i}=\left[\begin{array}{c}
\cos \alpha \\
\sin \alpha e^{j \psi}
\end{array}\right] \begin{array}{l}
0 \leq \alpha \leq \frac{\pi}{2} \\
-\pi \leq \psi \leq \pi
\end{array}
\end{gathered}
$$

where $\underline{\mathbf{w}}_{i}$ is the optimum channel that leads to increase the number of pixel candidates with respect to the single-channel data. The principle goal of this step is to find optimum projection vector for each pixel of SAR images based on certain pixel selection criteria. Parametrization of this vector lead to reduce the issue to find four defined angle parameters, i.e. $(\alpha, \beta, \delta, \gamma)$ for quad-pol data and $(\alpha, \psi)$ for dual-pol data with determined ranges. Notice that to solve this problem, equal scattering mechanisms (ESMs) constraint is applied in order to avoid changing in the scatterer phase center from interferogram to interferogram [13], [14], i.e., $\left(\underline{\mathbf{w}}_{1}=\underline{\mathbf{w}}_{2}=\cdots=\underline{\mathbf{w}}_{n}\right)$. Hence projection vector for each pixel must be preserved along all generated interferograms. In fact, we search whole of polarimetric space to optimize selection criteria and select the most stable scattering mechanism over the time of data acquisitions. By this basic formulation, we can introduce the two most important selection criteria for PS detection.

\section{A. Average Coherence}

The coherence optimization is considered as a selection criterion in which the optimum projection vector is a vector that maximize the mean value of interferometric coherence. High average coherence is a common indicator to evaluate phase quality. Pixels with higher mean coherence $\overline{|\gamma|}$ than a given threshold are selected as PSs.

$$
\overline{|\gamma|}=\frac{1}{N} \sum_{k=1}^{N}\left|\gamma_{k}\right| .
$$

1) Best: the differential phase quality can be improved by using a simple way that operates based on choosing the polarimetric channel with the maximum temporally averaged mean coherence, i.e.,

$$
\left|\gamma_{\text {Best }}\right|=\max \left\{\overline{\left|\gamma_{h h}\right|}, \overline{\left|\gamma_{h v}\right|}, \overline{\left|\gamma_{v v}\right|}\right\}
$$

This method is first introduced in [11].

2) ESM-MB: the most conventional method to optimize the phase quality of interferograms is to acquire the projection vector that maximizes the generalized polarimetric interferometric coherence which is defined in [12] as

$$
\gamma_{k}=\frac{\underline{\mathbf{w}}^{H}\left[\boldsymbol{\Omega}_{12}\right] \underline{\mathbf{w}}}{\sqrt{\underline{\mathbf{w}}^{H}\left[\mathbf{T}_{11}\right] \underline{\mathbf{w}}} \sqrt{\underline{\mathbf{w}}^{H}\left[\mathbf{T}_{22}\right] \underline{\mathbf{w}}}} .
$$

where subscript $k$ denotes the $k$ th interferogram, $\left[\mathbf{T}_{11}\right],\left[\mathbf{T}_{22}\right]$ are individual coherency matrices of each PolSAR data set, $\left[\boldsymbol{\Omega}_{12}\right]$ is the polarimetric interferometric coherency matrix. In fact, the problem in this case is to search whole polarimetric space to find $\underline{\mathbf{w}}$ that provides maximum average coherence for each pixel in the images. After projecting the data onto the acquired optimum vector for each pixel, the average coherence is calculated and points with higher value than the pre-defined threshold will be chooses as PSs. Coherence optimization technique using parametrization of $\underline{\mathbf{w}}$ in (4) and (5) is possible, but the main drawback is its high computational cost. In this study, we apply the approach proposed in [14] refered as the multi-baseline ESM shortly named ESM-MB to find the optimum $\mathbf{w}$ which is an analytical way based on eigenvalue problem.

3) Mean Intensity Polarimetric Optimization (MIPO): Another appropriate method to improve the coherence was proposed in [11] in which the projection vector is defined based on optimizing the average intensity over a stack of SAR images for each pixel. In polarimetric case, intensity of projected signal can be written as:

$$
\begin{gathered}
I_{\text {projected signal }}=|\mu|^{2}=\mu \cdot \mu^{*} \\
\bar{I}=\mathrm{E}\left\{\mu \cdot \mu^{*}\right\}=\underline{\mathbf{w}}^{H} \widehat{\widehat{\mathbf{T}}} \underline{\mathbf{w}}_{i}
\end{gathered}
$$

where $E\{\cdot\}$ is the expectation operator. $\widehat{\mathbf{T}}$ is the average of coherency matrices and is Hermitian semidefinite positive. The objective function is to maximize the average intensity for a given pixel. This can be solved as an eigen-value problem. $\underline{\mathbf{w}}$ is the corresponding eigenvector to the maximum eigenvalue of $\widehat{\mathbf{T}}$ for each pixel. By the optimum scattering mechanism, the optimized data is produced with projecting Pauli vector into the optimum vector and the coherence criterion is then used on new generated data.

\section{B. $A D I$}

Another estimator to select PSs along a stack of SAR images is ADI which is formulated in single-channel case as

$$
D_{a}=\frac{\sigma_{a}}{\bar{a}}
$$

For the case of quad-pol data, we apply several useful techniques to find optimum channel, the same as average coherence above. This provides the minimum $D_{a}$ in the polarimetric space in order to increase the number of PSs and improve performance of PSI.

1) Best: The first and simple way to enhance the differential phase quality, in the other words, improve the $D_{a}$ includes in selecting the polarimetric channel that leads to the lowest value of $D_{a}$ along the stack of PolSAR data images. This is similar to Best for the average coherence estimator and is formulated as follows for each pixel:

$$
D_{a, B e s t}=\min \left\{D_{a, h h}, D_{a, h v}, D_{a, v v}\right\}
$$


2) $E S M-M B$ : In polarimetric case, $D_{a}$ can be retrieved with replaceing amplitude of $S$ of all data sets by polarimetric scattering coefficient $\mu$, under the ESM assumption for all acquisitions. In this case, we have

$$
D_{a}=\frac{\sigma_{a}}{\bar{a}}=\frac{1}{\overline{\left|\underline{\mathbf{w}}^{H} \underline{k}\right|} \sqrt{N}} \sqrt{\sum_{i=1}^{N}\left(\overline{\left|\underline{\mathbf{w}}^{H} \underline{k}_{i}\right|}-{\overline{\left|\underline{\mathbf{w}}^{H} \underline{k}\right|}}^{2} .\right.} .
$$

This estimator is exploited to evaluate the phase stability of scatterers. Pixels with lower value than the given threshold are selected as PSs. In polarimetric case, search is done through whole polarimetric space to find best projecting vector $\underline{\mathbf{w}}$ that minimizes $D_{a}$ estimator. In this paper, we apply a beneficial and common metaheuristic search approach named as Particle Swarm Optimization (PSO) [15] to find optimum scattering mechanism for each pixel, In fact, the objective function is to minimize value of (II-B2) for each pixel based on four known parameters with finite ranges. The metaheuristic-search approach will find the values of $(\alpha, \beta, \delta, \gamma)$ in quad-pol case or $(\alpha, \psi)$ in dual-pol case that minimize ADI. Its initial values are determined based on a coarse grid search with step size of $15^{\circ}$ for all of angles. Performance of the PSO method is investigated in evaluation of optimum channel and detection of coherent scatterers over different cases of polarimetric capability, i.e., dual- and quad-pol cases.

3) MIPO: This method was proposed in [11]. The projection vector $\underline{\mathbf{w}}$ is defined as explained in the coherence estimator, based on optimization of the average intensity over a stack of SAR images for each pixel, with the same assumption in the coherence case.

\section{DATA SeTS}

The data used in this study were collected by the JPL/UAVSAR's UAVSAR, which is an L-band airborne polarimetric repeat-pass interferometric radar system. The study area is Sacramento-San Joaquin Delta region, CA, consisting of buildings, roads, river, parks, agricultural land. A total of 15 sequential acquisitions cover a time interval of two years, i.e., from Novermber 2014 to October 2016. The quad-pol SLC data in radar geometry are downloaded from the JPL UAVSAR data portal. Fig. 1 shows the average Pauli RGB composite of the scenes.

\section{RESULTS}

\section{A. Average Coherence Results}

The performance of different approaches for coherence optimization are compared in terms of PSs' quantity. A multilook of $5 \times 5$ pixels is applied to compute the mean coherence. Fig. 2(a) represents the histogram of average coherence $(\mid \overline{\gamma \mid})$ obtained for different polarimetric channels $(\mathrm{HH}, \mathrm{HV}, \mathrm{VV}$, and Pauli channels) and for the optimum channel obtained by quad-pol data and the number of PSs selected for different methods is summarized in Fig. 2(b).

Notice that the number of selected pixels is increased for three optimized data compared to the single-pol data. For the ESM-MB case, a dramatic improvement with respect

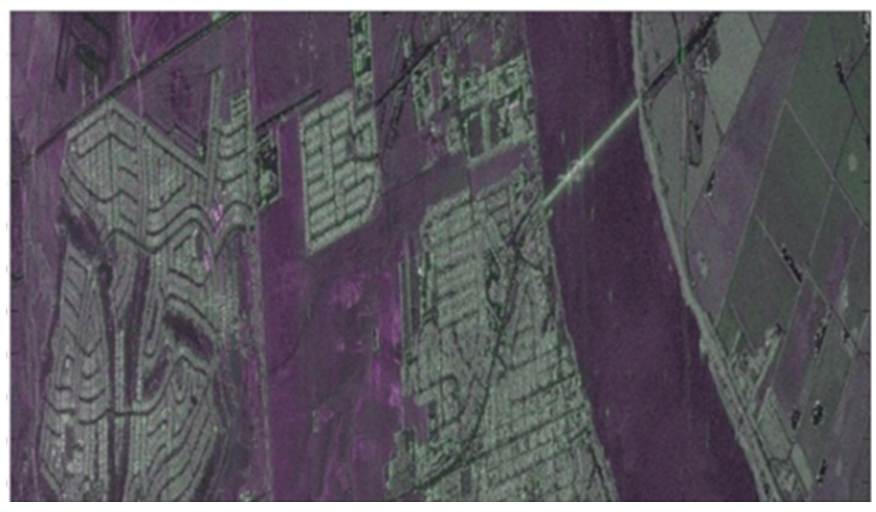

Fig. 1. The average Pauli RGB composite ( $\mathrm{R}=\mathrm{HH}-\mathrm{VV}, \mathrm{G}=\mathrm{HV}, \mathrm{B}=\mathrm{HH}+\mathrm{VV})$ of the quad-polarimetric UAVSAR acquisitions.
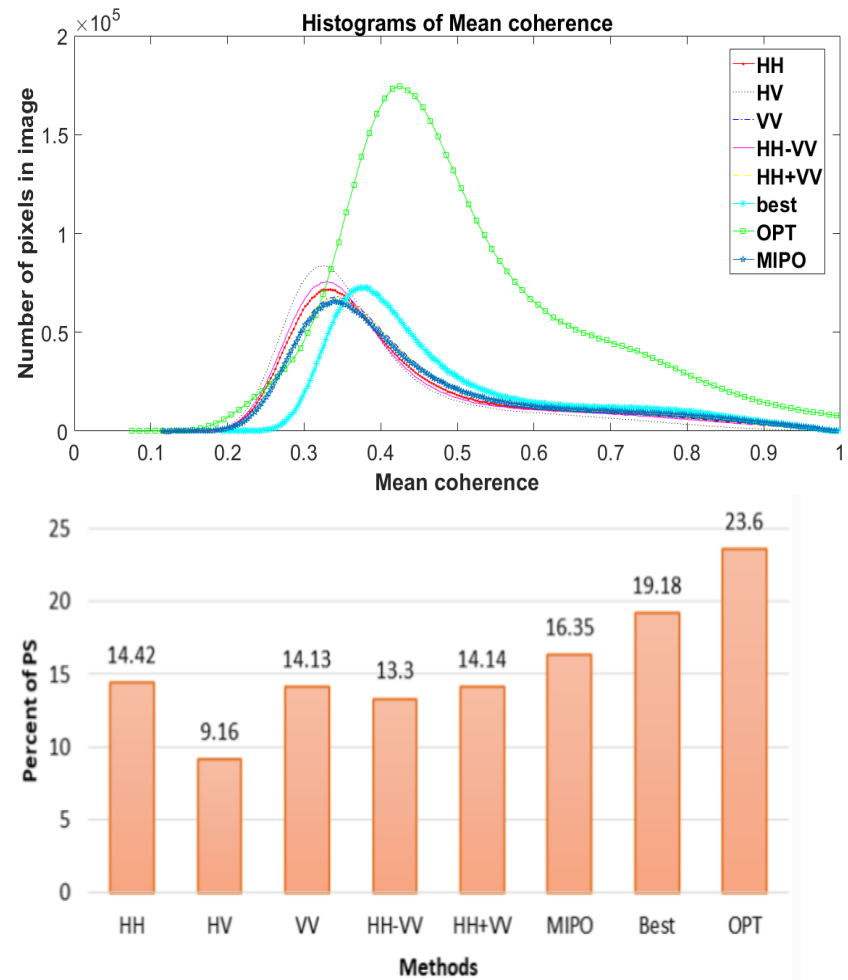

Fig. 2. (Top) histograms of $\overline{|\gamma|}$ for channels HH, HV, VV, Pauli and for the Best, MIPO, and ESM coherence stability optimization methods. (Bottom) detected PSs using different channels in the coherence estimator.

to single HH channel (about 63\%) is achieved with higher computational load. The MIPO and Best methods perform better than the HH channel (only) with an increment of about $15 \%$ and $33 \%$, respectively, and perform slightly worser than the EMS-MB method. Accessibility to polarimetric data are led to have improvements in the number of pixel selection and ESM-MB method implements as the best optimum method regardless of the high computational cost.

\section{B. ADI Results}

For the ADI estimator, only those pixels with $D a<0.3$ are selected. In different polarization configurations (quad-po 

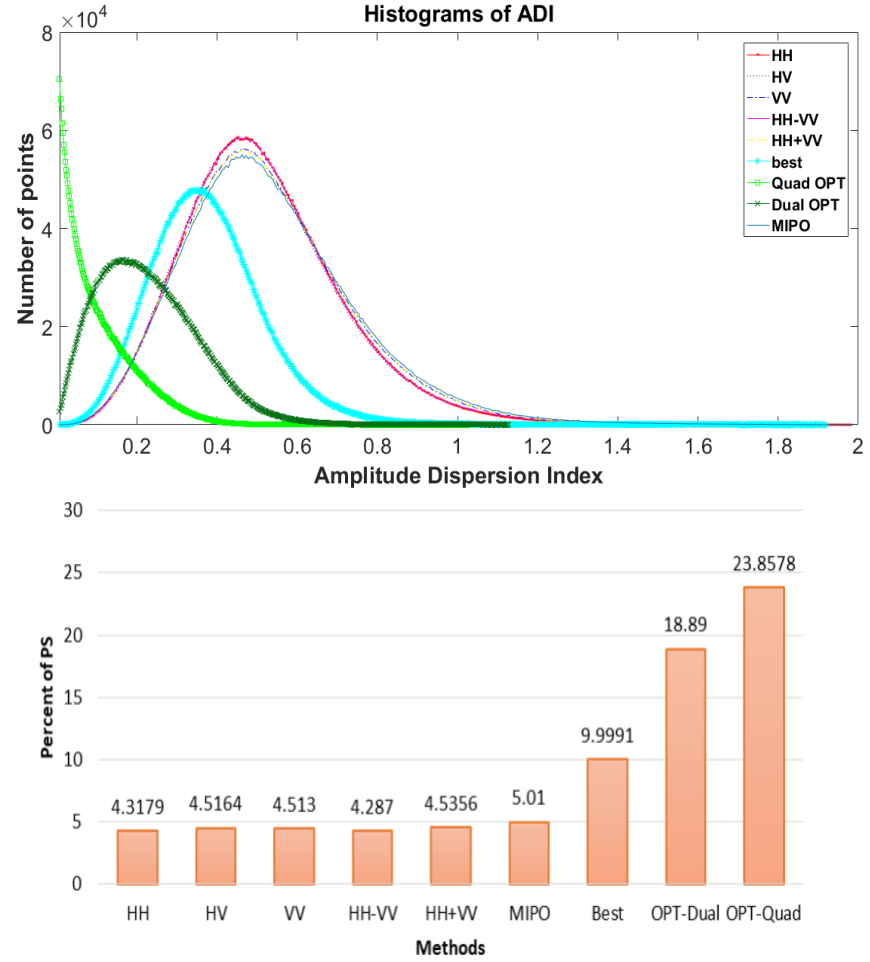

Fig. 3. (Top) histograms of ADI for channels HH, HV, VV, Pauli and for the Best, MIPO, and ESM. (Bottom) detected PSs using different channels in ADI estimator.

and dual-pol: HH/VV), we test the PSO search approach to find optimum $\underline{\mathbf{w}}$ in the ESM-MB method. ESM-MB provides the best performance in terms of PS density and quantity, in comparison with other approaches. For quad-pol images, MIPO method shows an improvement about $16 \%$ compared to HH. Best algorithm implements better than MIPO approach and reaches an increase of $130 \%$ compared with $\mathrm{HH}$ channel. There is a significant improvement for the ESM-MB approach, as expected, of about $450 \%$ (6 times more PSs).

Fig. 3(a) demonstrates the histogram of ADI obtained for different polarimetric channels (HH, HV, VV, Pauli channels) and for the optimum channel obtained by dual-pol and quadpol cases. The amplitude stability is significantly improved when using the ESM-MB optimized by PSO for quad pol channel. In particular, the ESM-MB for quad-pol, plotted as light green curve, produces the lowest ADI values and then for the dual-pol case, plotted as dark green curve. The number of PSs selected for different channels are summarized in Fig. 3(b)

\section{Discussion AND CONCLUSION}

In this paper, the most common approaches of polarimetric optimization were evaluated and considered for stack of UAVSAR quad-pol data to enhance the phase stability of the interferograms. Therefore the improvement of phase quality leads to increase the number of pixel candidates. In fact, this increment provided more information to have denser and valid results for deformation maps. Two pixel selection criteria are considered, mean coherency and amplitude dispersion index. There are several methods to optimize each criterion. We analyze the most common and operational optimization methods. For two criteria, the so-called Best is the simplest method with proper computational cost time. In the MIPO approach, the polarimetric channel that maximizes the mean intensity of data over time is achieved based on eigen problem. This is one of the estimators applied over the intensity optimized data to select PSs. The ESM-MB approach provides larger improvement in both estimators. In the coherence estimator, due to computation load of the numerical solution, we tested analytical solution to find the optimum projection vector for each pixel. In ADI, due to lack of an analytical approach, we applied a numerical oprimization technique named PSO for optimizing the projection vector in the ESM-MB method.

\section{REFERENCES}

[1] Z. Li, W. Feng, Z. Xu, P. Cross, and J. Zhang, "The 1998 mw 5.7 zhangbei-shangyi (china) earthquake revisited: A buried thrust fault revealed with interferometric synthetic aperture radar," Geochemistry, Geophysics, Geosystems, vol. 9, no. 4, pp. n/a-n/a, 2008, q04026.

[2] O.-I. Kwoun, Z. Lu, C. Neal, and C. Wicks, "Quiescent deformation of the aniakchak caldera, alaska, mapped by insar," Geology, vol. 34, no. 1, pp. 5-8, 2006.

[3] Y. Fialko, "Interseismic strain accumulation and the earthquake potential on the southern san andreas fault system," Nature, vol. 441, no. 7096, p. $968,2006$.

[4] S. Samsonov, K. F. Tiampo, and J. B. Rundle, "Application of dinsargps optimization for derivation of three-dimensional surface motion of the southern california region along the san andreas fault," Computers and Geosciences, vol. 34, no. 5, pp. 503-514, 2008.

[5] V. Akbari and M. Motagh, "Improved ground subsidence monitoring using small baseline SAR interferograms and a weighted least squares inversion algorithm," IEEE Geoscience and Remote Sensing Letters, vol. 9, no. 3, pp. 437-441, 2012.

[6] R. Iglesias, D. Monells, X. Fabregas, J. J. Mallorqui, A. Aguasca, and C. Lopez-Martinez, "Phase quality optimization in polarimetric differential SAR interferometry," IEEE Trans. Geosci. Remote Sens., vol. 52, no. 5, pp. 2875-2888, 2014.

[7] A. Ferretti, C. Prati, and F. Rocca, "Permanent scatterers in sar interferometry," IEEE Trans. Geosci. Remote Sens., vol. 39, no. 1, pp. 8-20, 2001.

[8] P. Berardino, G. Fornaro, R. Lanari, and E. Sansosti, "A new algorithm for surface deformation monitoring based on small baseline differential sar interferograms," IEEE Trans. Geosci. Remote Sens., vol. 40, no. 11, pp. 2375-2383, 2002.

[9] O. Mora, J. J. Mallorqui, and A. Broquetas, "Linear and nonlinear terrain deformation maps from a reduced set of interferometric sar images," IEEE Transactions on Geoscience and Remote Sensing, vol. 41, no. 10, pp. 2243-2253, 2003.

[10] A. Ferretti, C. Prati, and F. Rocca, "Nonlinear subsidence rate estimation using permanent scatterers in differential SAR interferometry," IEEE Trans. Geosci. Remote Sens., no. 5, pp. 2202-2212, 2000.

[11] V. D. Navarro-Sanchez, J. M. Lopez-Sanchez, and L. Ferro-Famil, "Polarimetric approaches for persistent scatterers interferometry," IEEE Trans. Geosci. Remote Sens., vol. 52, no. 3, pp. 1667-1676, 2014.

[12] S. R. Cloude and K. P. Papathanassiou, "Polarimetric SAR interferometry," IEEE Trans. Geosci. Remote Sens., vol. 36, no. 5, pp. 1551-1565, 1998.

[13] V. D. Navarro-Sanchez, J. M. Lopez-Sanchez, and F. Vicente-Guijalba, "A contribution of polarimetry to satellite differential SAR interferometry: Increasing the number of pixel candidates," IEEE Geoscience and Remote Sensing Letters, vol. 7, no. 2, pp. 276-280, 2010.

[14] M. Neumann, L. Ferro-Famil, and A. Reigber, "Multibaseline polarimetric SAR interferometry coherence optimization," IEEE Geoscience and Remote Sensing Letters, vol. 5, no. 1, pp. 93-97, 2008.

[15] X.-S. Yang, Engineering optimization: an introduction with metaheuristic applications. John Wiley \& Sons, 2010. 